\title{
Reflets
}

Revue ontaroise d'intervention sociale et communautaire

\section{Pour exprimer son histoire en français}

\section{Marie-Luce Garceau}

Volume 7, numéro 2, automne 2001

Le travail social en Ontario

URI : https://id.erudit.org/iderudit/026362ar

DOI : https://doi.org/10.7202/026362ar

Aller au sommaire du numéro

Éditeur(s)

Reflets : Revue ontaroise d'intervention sociale et communautaire

ISSN

1203-4576 (imprimé)

1712-8498 (numérique)

Découvrir la revue

Citer cet article

Garceau, M.-L. (2001). Pour exprimer son histoire en français. Reflets, 7(2), 145-150. https://doi.org/10.7202/026362ar

Tous droits réservés (C) Reflets : Revue ontaroise d'intervention sociale et communautaire, 2001

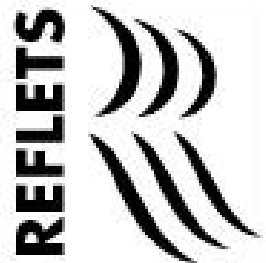

Ce document est protégé par la loi sur le droit d'auteur. L'utilisation des services d'Érudit (y compris la reproduction) est assujettie à sa politique d'utilisation que vous pouvez consulter en ligne.

https://apropos.erudit.org/fr/usagers/politique-dutilisation/ 


\section{Pour exprimer son histoire en français}

par

Marie-Luce Garceau, professeure, École de service social, Université Laurentienne

Ironiquement, ce court article pourrait tout aussi bien s'intituler Cent fois sur le métier, mesdames, remettez votre ouvrage. En effet, depuis les dernières années, les organismes de femmes francophones de toute la province ne cessent de réitérer aux gouvernants et aux fonctionnaires de l'Ontario, leurs besoins de services en français en matière de violence contre les femmes. La multitude de recherches provenant de tous les coins de la province est telle que nous nous demandons si, un jour, nos dirigeants entendront vraiment ce que les femmes ont à dire sur leurs besoins et les services qu'elles souhaitent ardemment voir en place ${ }^{1}$.Voici donc, une fois de plus, un document qui s'ajoute à longue liste de ceux parvenus dans les hautes sphères gouvernementales.

Pour exprimer son histoire en français ${ }^{2}$ est une recherche-action sur les besoins, les services et les modèles de prestation de services en matière de violence en français dans le Nord de l'Ontario. Ce document présente les résultats d'une recherche-action qualitative, menée par le Centre des femmes francophones du Nord-Ouest de l'Ontario et ses partenaires, le Centre Victoria pour femmes de Sudbury et l'Action ontarienne contre la violence faite aux femmes. Le but du projet a été d'effectuer une recherche-action auprès des intervenantes offrant des services en matière de violence aux femmes francophones du Nord-Ouest, de la Municipalité régionale de Sudbury et de l'Algoma. Elle comportait deux volets principaux. Le premier volet a servi à identifier les besoins et les 
services à mettre en place dans ces régions. Pour ce faire, cinquante (50) entrevues ont été effectuées auprès d'informatrices clés des trois régions. Le second volet a permis d'explorer, en collaboration avec les femmes des différents milieux, des modèles de prestation de services en matière de violence faite aux femmes qui soient représentatifs des diverses réalités des communautés francophones de ces trois régions. Pour ce faire, sept rencontres de groupes de discussion ont été menées dans les trois régions mentionnées. Et, après une analyse qualitative des résultats, l'équipe de chercheures est retournée dans les différentes régions pour valider son analyse et confirmer les résultats.

\section{Démarche du projet}

Les entrevues auprès des informatrices clés ont permis de vérifier leurs perceptions de l'ensemble des besoins des femmes victimes de violence conjugale, d'agressions à caractère sexuel ou d'autres formes d'abus et les services qui sont actuellement en place pour y répondre. Puis, il s'est agi de vérifier quels services doivent être développés et d'identifier des pistes de partenariats ou d'ententes pouvant permettre de servir adéquatement les femmes francophones victimes de violence, d'agression ou d'autres formes d'abus, dans les régions du Nord-Ouest, de l'Algoma, de la Municipalité régionale de Sudbury et de Sudbury-Est.

Les groupes de discussion ont tablé sur les réflexions des informatrices clés comme une sorte de tremplin permettant aux groupes de femmes de diverses localités de réfléchir collectivement sur les besoins, les priorités dans les services à mettre en place et sur le développement d'un modèle de prestation de services qui refléterait le mieux leur propre localité. Quant aux besoins, les participantes ont confirmé les besoins des femmes violentées identifiés, au préalable, par les informatrices clés. De plus, un besoin appelle sa satisfaction par la mise en place de services. C'est pourquoi les groupes de discussion ont aussi identifié certaines 
priorités dans les services à mettre en place. Pour l'essentiel, nous les présentons brièvement parce qu'ils sont au fondement du modèle de prestations de services qui a été développé dans chacune des localités :

- Accompagnement

- Lieu d'hébergement

- Lieu de réflexion

- Lieu anonyme et confidentiel

- Sensibilisation et prévention

- Connaissances sur les droits des femmes

- Intervention de crise à court et long terme et de groupe

- Programmes sur l'estime de soi ou axés sur les habiletés de vie

- Formation pour les intervenantes

- Concertation entre les services et les programmes

- Augmentation du nombre d'intervenantes

- Permanence des organismes francophones

- Services aux enfants qui ont vécu de la violence

- Amélioration du suivi judiciaire et des services de police

- Dépistage de la violence

- Aide financière directe et aide à la création d'emploi

- Formation des professionnels de tous les milieux

- Service d'aide aux femmes dans les communautés éloignées

- Soutien et entraide

Quant aux modèles de prestation de services en violence, les chercheures ont présenté trois modèles idéaux afin que les groupes puissent en discuter et développer celui qui conviendrait le mieux aux besoins des femmes. Pour l'essentiel, nous présentons un abrégé de ces modèles à la page suivante.

Dans les discussions, les participantes n'ont pas mis les modèles en opposition. Premièrement, elles ont tenté de combler les espaces qui les séparaient et d'intégrer des aspects d'un modèle à l'autre. Deuxièmement, elles ont émis l'idée qu'il y avait une gradation d'un modèle à l'autre et que leur objectif à long terme était l'extension des services vers la gamme complète. Troisièmement, elles indiquent que pour les femmes de petites localités ou de milieux ruraux isolés, il importe qu'elles aient aussi accès à une 
forme d'hébergement sécuritaire. Quatrièmement, elles avancent l'idée qu'offrir des services en français en violence ne constitue pas un dédoublement de services. Cinquièmement, elles refusent de scinder la réalité des femmes violentées en une multitude de problématiques séparées les unes des autres. Plutôt, elles souhaitent une porte d'entrée, un guichet unique qui permettrait de servir les femmes dans toutes les facettes de la vie des femmes. Finalement, elles insistent sur l'importance d'un suivi offert 24 heures par jour par la ligne de crise.

\begin{tabular}{lll}
\hline $\begin{array}{l}\text { Centre de mieux-être } \\
\text { Pour les femmes }\end{array}$ & $\begin{array}{l}\text { Centre multiservices } \\
\text { (guichet unique) }\end{array}$ & $\begin{array}{l}\text { Refuge à court et moyen } \\
\text { terme }\end{array}$ \\
\hline $\begin{array}{l}\text { Intervention sur des problèmes } \\
\text { multiples. }\end{array}$ & $\begin{array}{l}\text { Offre locale de services } \\
\text { communautaires } \\
\text { Axé de réflexion }\end{array}$ & $\begin{array}{l}\text { Offre d'hébergement à proximité } \\
\text { du lieu de résidence } \\
\text { Milieu francophone de langue et } \\
\text { de culture }\end{array}$ \\
$\begin{array}{l}\text { Offre d'intervention de groupe, } \\
\text { soutien et accompagnement }\end{array}$ & $\begin{array}{l}\text { Équipes d'intervenantes qui peuvent } \\
\text { se déplacer vers les communautés } \\
\text { Service de références aux } \\
\text { organismes et services }\end{array}$ & $\begin{array}{l}\text { Assure le traitement à court et à } \\
\text { moyen terme. }\end{array}$ \\
$\begin{array}{l}\text { Service de références aux } \\
\text { organismes et services Centre } \\
\text { d'information sur les ressources. } \\
\text { organismes et les agences locales au }\end{array}$ & $\begin{array}{l}\text { Centre d'information sur les } \\
\text { ressources }\end{array}$ \\
$\begin{array}{l}\text { Approche féministe. } \\
\text { Assure confidentialité et anonymat. } \\
\text { Prévention et sensibilisation } \\
\text { communautaire. }\end{array}$ & $\begin{array}{l}\text { Assure confidentialité et anonymat } \\
\text { Éqiupe volante en prévention et } \\
\text { sensibilisation communautaire }\end{array}$ & $\begin{array}{l}\text { Approche féministe } \\
\text { Assure confidentialité et anonymat } \\
\text { Prévention et sensibilisation } \\
\text { communautaire }\end{array}$ \\
\hline
\end{tabular}

\section{Recommandations des groupes de discussion}

À partir des discussions qui ont eu lieu dans toutes les localités, les femmes ont mis de l'avant certaines recommandations pour contrer la violence conjugale, les agressions à caractère sexuel ou toutes autres formes d'abus dont les femmes sont victimes. 
- Que les gouvernements assurent la mise en place, la permanence financière et les ressources humaines adéquates d'un Centre de mieux-être francophone pour les femmes violentées de Thunder Bay.

- Que les gouvernements assurent la mise en place, la permanence financière et les ressources humaines adéquates d'un Centre multiservices francophone pour les femmes violentées de Geraldton, de Longlac et de Nakina.

- Que les gouvernements assurent la mise en place, la permanence financière et les ressources humaines adéquates d'un Refuge francophone à court et moyen termes pour les femmes violentées de Sudbury.

- Que les gouvernements assurent la mise en place, la permanence financière et les ressources humaines adéquates d'un Centre multiservices francophone pour les femmes violentées de Saint-Charles.

- Que les gouvernements assurent la mise en place, la permanence financière et les ressources humaines adéquates d'un Centre multiservices francophone pour les femmes violentées de Sault Sainte-Marie.

- Que les gouvernements assurent la mise en place, la permanence financière et les ressources humaines adéquates d'un Centre multiservices francophone pour les femmes violentées de Dubreuilville.

Attendu que la demande de services qui est exprimée dans les localités visitées est le reflet d'une situation généralisable à l'ensemble du Nord de la province, nous recommandons:

- Que les gouvernements assurent la mise en place de services en français dans le Nord de l'Ontario, qui soient accessibles, flexibles et complets en matière de violence faite aux femmes.

- Que les gouvernements assurent la permanence des services en français et les ressources humaines adéquates en matière de violence contre les femmes dans le Nord de l'Ontario.

En guise, de conclusion, nous n'avons qu'un seul souhait et vous l'aurez deviné. C'est que les femmes puissent enfin recevoir, dans leur langue et leur culture, l'ensemble de tous les services 
dont elles ont besoin et auxquels elles ont droit, afin de favoriser leur processus de guérison suite à des actes de violence qui sont inacceptables, intolérables.

\section{Notes}

1. Au cours des années, la revue Reflets a laissé une large place aux résultats des pratiques et des recherches féministes et aux travaux sur la violence contre les femmes et les enfants en Ontario. Tout particulièrement, on peut se référer au volume 2, numéro 1 (1996) et au volume 3, numéro 2 (1997).

2. Le présent article est un sommaire des résultats d'une recherche-action menée par le Centre des femmes du Nord-Ouest de l'Ontario et ses partenaires, le Centre Victoria pour femmes de Sudbury et l'Action ontarienne contre la violence faite aux femmes. Cette recherche-action a été subventionnée par Condition féminine Canada, Programme de promotion de la femme. Son co-auteur est Marc Charron, chargé d'enseignement à l'Université Laurentienne de Sudbury.

On peut se procurer une copie de Pour exprimer son histoire en français : recherche-action sur les besoins, les services et les modèles de prestation de services en français en matière de violence contre les femmes dans le Nord de l'Ontario en contactant le Centre des femmes francophones du NordOuest de l'Ontario à l'adresse suivante : C.P. 2041, Thunder Bay, Ontario, P7A 8A9. On peut aussi joindre le CFFNOO par téléphone au (705) 346-1210, par télécopieur au (705) 346-1220 ou par courriel à cffnoo@afnoo.org 